\title{
Boundary-Layer Receptivity and Integrated Transition Prediction
}

\author{
Chau-Lyan Chang* and Meelan Choudhari ${ }^{1}$ \\ NASA Langley Research Center, Hampton, VA23681
}

\begin{abstract}
The adjoint parabolized stability equations (PSE) formulation is used to calculate the boundary layer receptivity to localized surface roughness and suction for compressible boundary layers. Receptivity efficiency functions predicted by the adjoint PSE approach agree well with results based on other nonparallel methods including linearized Navier-Stokes equations for both Tollmien-Schlichting waves and crossflow instability in swept wing boundary layers. The receptivity efficiency function can be regarded as the Green's function to the disturbance amplitude evolution in a nonparallel (growing) boundary layer. Given the Fourier transformed geometry factor distribution along the chordwise direction, the linear disturbance amplitude evolution for a finite size, distributed nonuniformity can be computed by evaluating the integral effects of both disturbance generation and linear amplification. The synergistic approach via the linear adjoint PSE for receptivity and nonlinear PSE for disturbance evolution downstream of the leading edge forms the basis for an integrated transition prediction tool. Eventually, such physics-based, high fidelity prediction methods could simulate the transition process from the disturbance generation through the nonlinear breakdown in a holistic manner.
\end{abstract}

\section{Introduction}

$\mathrm{T}$ ransition prediction plays an important role in modern aerodynamic designs, especially, for supersonic and hypersonic vehicles. Laminar flow control (LFC) holds great promise for reducing the skin friction drag due to turbulent boundary layers over the vehicle surface. A successful laminar flow design can significantly reduce the drag and increase the vehicle performance. Recent Defense Advanced Research Projects Agency (DARPA) and NASA projects have been geared towards developing LFC technologies for next-generation supersonic vehicles. Physics-based transition prediction is an essential building block for successful implementation of such techniques. Depending on the extent of the flow physics to be simulated (which, in turn, is determined by a combination of required accuracy and turnaround times), several prediction methods may be used. At low fidelity, the linear stability based $\mathrm{N}$-factor method is used to assess a design concept, or in many cases, even serve as the sole prediction tool for design. At high fidelity, several alternatives exist to simulate the relevant stages of the intricate laminar turbulent transition process, from receptivity to the nonlinear breakdown to turbulence. An integrated transition prediction methodology thus consists of a hierarchical yet complementary set of prediction tools tailored to accurately simulate the flow physics involved at various stages.

At NASA Langley Research Center, recent transition prediction related works have focused on the development of a physics-based, integrated transition prediction software tool box that includes a range of methods from the conventional N-factor correlation (based on either the linear stability theory (LST) or the more advanced parabolized stability equations (PSE) approach) to simulations based on the absolute disturbance amplitudes. The Langley Stability and Transition Analysis Codes (LASTRAC) software represents the first release of the integrated tool development [1]. The code can be used for transition-related efforts for two-dimensional (2D) or infinite swept wing configurations. LASTRAC Version 2.0 extends the prediction methods for general three-dimensional boundary layers [2].

Despite the popularity of the $\mathrm{N}$-factor method for transition prediction, many design problems require a higher fidelity prediction method capable of simulating the finite amplitude of disturbances. One example is the LFC technique based on the spanwise periodic roughness elements (SPRE) [3]. In this innovative control concept,

\footnotetext{
• Research Scientist, Computational, Modeling, and Simulation Branch, email: Chau-Lyan.Chang@nasa.gov

Senior member, AIAA

${ }^{1}$ Research Scientist, Computational, Modeling, and Simulation Branch, Associate Fellow, AIAA
} 
spanwise roughness elements are introduced near the leading edge in order to artificially trigger early amplification of a less dominant stationary crossflow disturbance. Nonlinear interaction among the triggered and other modes delays or, in some cases, nearly suppresses the growth of the linearly highly unstable crossflow disturbances. In simulating such flow configurations, receptivity of the roughness elements and nonlinear evolution of the induced disturbance modes represent two key elements for accurate predictions. In Ref.[4], nonlinear PSE was used to perform preliminary parametric studies for the SPRE control of a supersonic infinite swept wing boundary layer. A range of initial amplitudes of the control and other unstable stationary or traveling crossflow modes was used to demonstrate possible scenarios for nonlinear mode competition in the presence of a relatively large amplitude control mode. However, the receptivity process that determines the initial amplitudes was only briefly discussed.

In a benign unsteady environment, stationary crossflow modes can be excited rather effectively via short-scale variations in surface geometry and surface suction (Choudhari and Streett [5]). When the amplitude of the wall variation is sufficiently small, linearized equations of motion may be used to predict the amplitudes of the generated crossflow instability wave (see refs.[6-7]). Nonlinear effects may become important when the forcing amplitude is sufficiently large. Following the pioneering works by Goldstein[8] and Ruban[9] using high Reynolds number asymptotic theories, much of the theoretical work was focused on the finite Reynolds number extension of the original framework constructed based on the inhomogeneous solutions of the classical linear stability theory (e.g. refs.[6-7]). In these analyses, the flow is assumed to be locally parallel; therefore, a higher fidelity method is necessary for stability problems with significant nonparallel effects (e.g., near the lower branch of a swept wing boundary layer).

The receptivity process in the absence of strong nonlinear effects can be completely described by solutions of a direct numerical simulation (DNS) or linear Navier-Stokes (LNS) equations solver. Such high fidelity simulations retain all the flow physics but are too expansive to be adopted as a routine design tool. Alternative approximate numerical solutions are also available. Prompted by design and optimization needs, the adjoint method has recently emerged as a viable tool for obtaining sensitivity derivatives encountered in may engineering applications. Tumin and Fedorov [18] and Hill[10] formulated the receptivity problem using the adjoint system of equations derived from the quasi-parallel stability equations. It was shown that the adjoint formulation gives solutions that agree with the asymptotic or finite Reynolds number theories. To account for nonparallel effects, the adjoint PSE approach has also been employed for incompressible boundary layers by Herbert [11], Airiau et al.[12], and Collis and Dobrinsky [13]. Bertolotti [14] used a combination of Fourier transform and Taylor series expansion to account for the nonparallel terms in the receptivity formulation for a growing boundary layer. Janke [17] investigated receptivity and nonlinear development of stationary crossflow instability for several incompressible boundary layer flows including the low-speed swept wing experiment at Arizona State University [19]. Streett [22] and Collis and Lele[16] have demonstrated the application of linearized Navier-Stokes equations in the context of crossflow receptivity in incompressible and subsonic boundary layers, respectively. Jiang et al. [20-21] have presented direct simulations of roughness-induced excitation of both crossflow and Gortler modes in supersonic and hypersonic boundary layers, respectively.

As mentioned previously, this paper is focused on the excitation of stationary crossflow disturbances via a spanwise periodic array of roughness elements or discrete suction holes because such configurations are being used in several LFC applications. Receptivity and nonlinear PSE calculations may be used in an integrated manner for parametric studies in these applications to ensure successful laminar flow design. Receptivity calculations also provide valuable insights with regard to optimal placement, spacing, and height of the roughness array. Here, this study assumes that the roughness height is small enough that any nonlinear surface disturbance effects are negligible. The adjoint linear PSE formulation is implemented as an additional module in the LASTRAC software towards the goal of seamless calculations of receptivity and subsequent disturbance evolution in compressible swept wing boundary layers. By further invoking the quasi-parallel assumption, the resulting adjoint LST formulation may also be used to obtain an equivalent of the finite Reynolds number theoretical solution. The next sections will discuss the formulation of the adjoint PSE method for localized receptivity; followed by treatment of distributed surface nonuniformities based on the calculated localized receptivity efficiency functions. Several test cases are presented to validate the methods and the computational module developed in this research. A case study for integrated transition prediction using the current receptivity approach is performed for LFC in a supersonic swept wing boundary layer. 


\section{Theoretical and Computational Approach}

For small amplitude surface disturbances, receptivity calculations associated with a localized nonuniformity provide the essential building block for predicting disturbance amplitudes due to arbitrary spatial distributions of surface nonuniformities [6]. This study only considers convective instabilities inside a boundary layer because the generation of absolute instabilities would require a completely different treatment. As mentioned previously, several theoretical methods such as the finite Reynolds number theory are available for calculating the receptivity of local nonuniformity. Within the framework of quasi-parallel linear stability theory, the adjoint approach provides an alternative formulation to the finite Reynolds number theory. To account for nonparallel effects, the adjoint method can be extended to the parabolized stability equations [11-13]. In principle, the adjoint system of the governing stability equations can be derived and integrated over a domain that includes the local nonuniformity of interest. Then, by simple algebraic rearrangements, the receptivity efficiency function can be related to the external forcing via simple integrals that involve the regular and adjoint eigensolutions of the governing system of equations. Most of the existing receptivity investigations using the adjoint approach were done for incompressible flows. Formulation of the adjoint approach for compressible boundary layer PSE may be derived by following similar procedures for the incompressible boundary layers [12-13].

Let $x, y$, and $\mathrm{z}$ denote the chordwise, wall-normal, and spanwise (or azimuthal for axisymmetric configurations) directions, respectively. The governing equations for the disturbance vector $\phi\left(\phi=\left(p^{\prime}, u^{\prime}, v^{\prime}, w^{\prime}, T^{\prime}\right)\right.$, representing pressure, velocities, and temperature perturbations) are

$$
\begin{aligned}
& \Gamma \frac{\partial \phi}{\partial t}+A \frac{\partial \phi}{\partial x}+B \frac{\partial \phi}{\partial y}+C \frac{\partial \phi}{\partial z}+D \phi=V_{x x} \frac{\partial^{2} \phi}{\partial x^{2}}+V_{x y} \frac{\partial^{2} \phi}{\partial x \partial y}+ \\
& V_{y y} \frac{\partial^{2} \phi}{\partial y^{2}}+V_{y z} \frac{\partial^{2} \phi}{\partial y \partial z}+V_{x z} \frac{\partial^{2} \phi}{\partial x \partial z}+V_{z z} \frac{\partial^{2} \phi}{\partial z^{2}}+f
\end{aligned}
$$

where $f$ represents the external forcing. For a 2D or infinite swept wing boundary layer, the disturbance vector of interest is periodic both in $t$ and $z$, with $\omega$ and $\beta$ representing temporal and spanwise wave numbers, respectively; i.e.,

$$
\phi(x, y, z, t)=\hat{\mathrm{A}} \tilde{\phi}(x, y) \exp \left(i \int_{\alpha_{0}} \alpha d \xi+\beta z-\omega t\right)
$$

The PSE method further assumes that the streamwise wave number, $\alpha$, is locally computed by adapting to the wave nature in the marching $(x)$ direction. This iteratively determined streamwise wave number not only alleviates the grid resolution required but also reduces the errors associated with the parabolizing assumption, a key ingredient of the PSE approach. Within the framework of linear theory, the disturbance amplitude $\hat{A}$ can be any constant. Substituting Eq. (2) into Eq. (1), and invoking usual assumptions underlying the parabolization of disturbance equations, the governing equations can be written in the following form:

$$
L \tilde{\phi}=\tilde{f}
$$

where $\tilde{f}=\iint f(z, t) \exp (i(\beta z-\omega t)) d z d t$ is the Fourier transform of the external disturbance forcing function and the PSE operator, $L$, is defined as

$$
L=\widetilde{A} \partial_{x}+\widetilde{B} \partial_{y}+\widetilde{D}-V_{y y} \partial_{y}
$$

For convenience, the equations have been scaled such that the coefficient matrix $V_{y y}$ involving purely viscous terms is independent of the wall-normal coordinate. The coefficient matrices, $\widetilde{A}, \widetilde{B}, \widetilde{D}$ in the above equation are functions of all wave numbers and the mean flow. Multiplying the left-hand side of Eq. (3) by a test vector function $\widetilde{\psi}$ and rearranging, the equation becomes

$$
\tilde{\psi} \cdot L \tilde{\phi}=\tilde{\phi} \cdot L^{*} \tilde{\psi}+\partial_{x} B_{x}+\partial_{y} B_{y}
$$


where the adjoint PSE operator and two additional terms on the right hand side are defined as:

$$
\begin{aligned}
& L^{*} \tilde{\psi}=\left[-\widetilde{A}^{T} \partial_{x}-\widetilde{B}^{T} \partial_{y}+\left(\widetilde{D}^{T}-\partial_{x} \widetilde{A}^{T}-\partial_{y} \widetilde{B}^{T}\right)-V_{y y} \partial_{y y}\right] \tilde{\psi} \\
& B_{x}=\widetilde{\phi} \cdot \widetilde{A}^{T} \widetilde{\psi} \\
& B_{y}=\widetilde{\phi} \cdot \widetilde{B}^{T} \widetilde{\psi}-\left(\partial_{y} \widetilde{\phi} \cdot V_{y y}^{T} \tilde{\psi}-\widetilde{\phi} \cdot V_{y y}^{T} \partial_{y} \tilde{\psi}\right)
\end{aligned}
$$

Let the test function $\widetilde{\psi}$ satisfy the adjoint parabolized equations, i.e.,

$$
L^{*} \tilde{\psi}=0
$$

In contrast to the original PSE, the adjoint PSE are marched from downstream to the upstream location where the forward PSE is initiated. Substituting Eqs.(5) and (9) into Eq. (3) yields

$$
\tilde{\psi} \cdot \tilde{f}=\partial_{x} B_{x}+\partial_{y} B_{y}
$$

In the absence of external forcing throughout the domain of interest, integration of Eq. (10) over a domain extending from $x=x_{i}$ to $x=x_{f}$ and $y=0$ to $y=\infty$ would lead to

$$
\int_{0}^{\infty}\left(\widetilde{\phi} \cdot \widetilde{A}^{T} \widetilde{\psi}\right) d y=\text { const }
$$

This adjoint constant provides a proper normalization to the adjoint solution during the backward marching. When external forcing and wall or free-stream nonuniformities are present, one may choose $x_{i}$ and $x_{f}$ to be located upstream and downstream of the nonuniformities. For nonzero forcing, similar integration of Eq. (10) yields the following equation for the disturbance amplitude (as defined in Eq. (2)) due to forcing contained within the range $\left(x_{i}, x_{f}\right)$ :

$$
\hat{A}\left(x_{f}\right)=\frac{\int_{0}^{\infty} \int_{x_{i}}^{x_{f}} \tilde{\psi} \cdot \widetilde{f} d x d y-\int_{x_{i}}^{x_{f}}\left(B_{y}(\infty)-B_{y}(0)\right) d x}{\left[\int_{b}^{\infty} \widetilde{\phi} \cdot \widetilde{A}^{T} \tilde{\psi} d y\right]_{x_{j}}}
$$

This equation relates the disturbance amplitude to any infield or boundary forcing via the regular and adjoint PSE shapefunctions. An analogous expression may be derived in the context of the adjoint LST equations by neglecting all nonparallel terms in Eqs. (3), (9) and (12).

From the standpoint of receptivity theory, it is more preferable to express the disturbance amplitude defined in Eq. (12) in terms of the efficiency function that relates the disturbance amplitude to the Fourier geometry factor due to the surface nonuniformity. Hence, Eq. (2) is rewritten in the following form:

$$
\phi(x, y, z ; \omega, \beta)=\varepsilon \hat{C}(x, \omega, \beta) \widetilde{\phi}(x, y) \exp \left(i \int_{\alpha_{0}} \alpha d \xi+\beta z-\omega t\right)
$$

where $\varepsilon$ is the amplitude of the surface nonuniformity. Within the framework of the quasi-parallel receptivity theory, the amplitude coefficient $\hat{C}$ can be further expressed as the product of a receptivity efficiency function and the geometry factor:

$$
\hat{C}=\Lambda(x, \omega, \beta) \widetilde{F}\left(\alpha_{r}, \beta\right)
$$

The efficiency function $\Lambda$ is independent of the geometry of the surface disturbance and depends only on the local profiles of the boundary layer mean flow. The geometry factor, $\widetilde{F}$, represents the Fourier coefficient of the wall nonuniformity evaluated at the wave number pair corresponding to the instability wave of interest. The decoupling 
of geometry and efficiency functions was first pointed out by Goldstein in his pioneering work of 1985[8]. Computing the efficiency function for a given mean flow and wave number thus provides a basis for further predicting the disturbance amplitude associated with a surface nonuniformity of finite spatial extent. In the present adjoint PSE approach, once the forward and backward marching solutions are computed, the efficiency function can be computed by using Eq. (12) locally at each marching location. Receptivity to various types of environmental forcing or surface nonuniformities may be treated similarly. This paper only deals with receptivity due to surface admittance and roughness. For example, if receptivity to wall blowing or suction is of interest, $\widetilde{\phi}(0)=\left(\widetilde{p}_{k}, 0, \widetilde{v}_{u}, 0,0\right)$ and the far field contribution to $B_{,}$vanishes. Similarly, a wall roughness may be treated as an equivalent forcing on the velocity perturbation vector by using the Taylor series expansion. Then the perturbation shapefunction vector becomes

$$
\widetilde{\phi}(0)=\left(\widetilde{p}_{w},-\widetilde{h}_{w} \partial U / \partial y,-\widetilde{h}_{w} \partial V / \partial y,-\widetilde{h}_{w} \partial W / \partial y, 0\right)
$$

where $(U, V, W)$ is the mean fiow velocity vector and $\tilde{h}_{w}$ is the iocal Fourier transformed geometry factor of the roughness element.

Numerical solutions of the efficiency function for a given nonuniformity thus consist of several steps. First, one must solve the regular PSE via a downstream marching until a desired downstream location is achieved. The adjoint PSE is then solved from that downstream location via an upstream adjoint marching until the initial chordwise location. The receptivity efficiency function distribution is then calculated using both the regular and adjoint PSE solutions together with the specified wall forcing using Eq. (12). The major advantage of the adjoint PSE approach is its efficiency compared to an LNS or DNS solver. The local receptivity efficiency functions, once obtained, may be used to predict disturbance amplitude for various types of geometry factors using the distributed receptivity formulation to be discussed in the next section.

When the surface nonuniformities span over a sufficiently large range comparable to the wavelength of the instability wave, the integral effects of the local nonuniformities must be evaluated. The procedure follows that given by Choudhari and Streett [6]. In the presence of distributed nonuniformities, effects of both disturbance generation and linear amplification must be accounted for in order to determine the disturbance amplitude evolution. This study considers a general roughness distribution described by $F(x, z)$ on the surface. For a spanwise wave number $\beta$, the geometry factor of the roughness element along the chordwise direction can be computed by the Fourier Transform:

$$
\widetilde{F}(x, \beta)=\int_{-\infty}^{\infty} F(x, z) e^{-i \beta z} d z
$$

Instead of Eq. (14), the amplitude coefficient for distributed receptivity can thus be expressed as:

$$
\begin{aligned}
& \hat{C}=\frac{1}{\sqrt{2 \pi}} \int_{\alpha_{0}} \Lambda(\xi, \beta) \widetilde{F}(\xi, \beta) \exp \left(-i \theta_{r}(\xi, \beta)\right) d \xi \\
& \theta_{r}(x, \beta)=\int_{\alpha_{0}}^{x} \alpha_{r}(\xi, \beta) d \xi
\end{aligned}
$$

In Eq. (16), the coefficient $1 / \sqrt{2 \pi}$ is not needed if the receptivity efficiency function is not scaled with $\sqrt{2 \pi}$. The chordwise wave number $\alpha$ in Eq. (17) refers to that of the instability wave and not the geometric characteristics of the wall roughness. Given the geometry of the roughness elements, the above two equations in conjunction with Eq. (13) can be used to calculate the amplitudes of the instability wave. Two aspects of the adjoint based PSE approach should be noted. Firstly, for a given configuration, the receptivity efficiency functions can be computed for a series of spanwise wave numbers (and frequencies for unsteady disturbances). These receptivity efficiency functions calculated can be used to determine disturbance amplitude for any given surface geometry. Secondly, the resolution required for the efficiency function calculation pertains to the mean flow (and thus efficiency function) variations. No clustering is necessary near the source of nonuniformities. The disturbance amplitude integration, Eqs. (13) and (16), can be performed with a completely different mesh in $x$. Proper clustering can also be added near the source region to improve the accuracy of amplitude predictions. 
Alternatively, one can also use direct numerical simulations for finite-size nonuniformities. This study used a previously developed DNS code [20-21] for receptivity calculations in this paper. The DNS code uses $6^{\text {th }}$ order compact differencing in streamwise and wall-normal directions and the same scheme or Fourier discretization in spanwise direction depending on whether or not the basic state is a function of the spanwise coordinate. The DNS results provide a means of validating the accuracy of the adjoint PSE method mentioned above. For receptivity calculations, the linear Navier-Stokes (LNS) approach (e.g. refs. [22-23]) is more efficient than the DNS. These direct solvers use the disk caching technique to solve a very large matrix formed by the high order discretization on a two-dimensional plane. With the state of the art CPU's, the turnaround time is pretty reasonable for the problem size pertinent to the receptivity calculations. However, in contrast to the efficiency function based prediction methods, calculations must be repeated when the geometry of the nonuniformities changes unless one uses the adjoint form of LNS equations as described in Ref. [13]. To improve the efficiency of the LNS formulation, this study also examines a new LNS formulation based on the existing PSE method. Let $L^{\prime}$ represent the discretized PSE operator which consists of the two discretized operators in $x$ and $y$ :

$$
L^{l}=L_{x}^{l}+L_{y}^{l}
$$

The streamwise derivatives in Eq. (18) are discretized by a first- or second-order backward differentiation and normal derivatives in $L_{y}$ are discretized with a fourth or higher order finite difference. The following equations are solved iteratively:

$$
\left(L^{l} \widetilde{\phi}\right)^{n+1}=\left(L_{x}^{l} \widetilde{\phi}\right)^{n}-\left(L_{x}^{h} \widetilde{\phi}\right)^{n}
$$

by multiple marching sweeps. The operator $L_{x}^{h}$ is a higher order version of $L_{x}^{l}$ in which the streamwise derivatives are discretized using either $4^{\text {th }}$ order central or $3^{\text {rd }}$ order upwind biased difference and the streamwise second derivative viscous terms are included. In contrast to the marching PSE operator, upstream influence is allowed in $L_{x}^{h}$. The left-hand side operator in Eq. (19) is the PSE operator and can be solved by a marching procedure from upstream to downstream. On the other hand, the right hand side is explicitly computed and added as a source term to the discretized PSE. If converged, Eq. (19) reduces to a high order linear Navier-Stokes equations, i.e.,

$$
\left(L_{x}^{h}+L_{y}^{l}\right) \widetilde{\phi}=0
$$

Numerical experiments indicate that a $3^{\text {rd }}$ order upwind biased scheme in $L_{x}^{h}$ is more robust than the $4^{\text {th }}$ order central scheme.

For a domain initiated by a specified shape function at the inlet, it takes only about $25-30$ iterations to converge the L2 norm to about $10^{-10}$. One advantage of this PSE-based LNS solver is the incorporation of the streamwise wave number $\alpha$ greatly reduces the grid resolution required in $x$ as compared to other physical space based LNS solvers. However, a number of receptivity problems involve a disturbance field with a broad spectrum, which implies that a unique $\alpha$ cannot be determined near the source region. In such cases, the spatial grid requirement becomes similar to the direct LNS solver, and therefore, the PSE-based LNS method may not yield significant savings in computational time. Nevertheless, it is still possible to incorporate a Fourier series expansion in $\alpha$ in the iterative solver to improve the solution accuracy without solving the full matrix. As the next section discusses, it is found that by setting the streamwise wave number to that of the instability wave of interest, one can still reduce the grid points required near the source region while adequately resolving the evolution of the eigenmode of interest. Continuous spectrum and other eigenmodes near the source region cannot be resolved using this approach unless sufficient grid points are used.

\section{Results and Discussion}

The adjoint PSE method for compressible boundary layers together with the disturbance amplitude integration for distributed surface nonuniformities have been implemented in the LASTRAC[1-2] code. For a given flow configuration, receptivity efficiency functions can be calculated for frequencies and spanwise wave numbers that are either most unstable or relevant to the disturbance environment. Once this "table" of efficiency function is established, the geometry factor obtained by processing the roughness or suction holes patterns on the surface can be used as an input for distributed receptivity calculations. Dominant instability modes from the receptivity calculations 
can be selected and analyzed further by using the nonlinear PSE method provided by the LASTRAC code. As an initial step, a loosely coupled approach of this type was deemed both more convenient and representative of the applications of interest.

The following discussions validate the computational methods outlined in the previous section by comparing the predicted localized receptivity efficiency functions with existing results in the literature. Adjoint PSE along with DNS or LNS results are cross-validated for some of the examples given below. For the Mach 2.4 supersonic swept wing case [3-4], transition analysis begins with prescribed roughness elements distribution along the spanwise and chordwise directions and ends with the absolute nonlinear amplitude evolution in the downstream region. In this way, transition predictions are performed in an integrated manner by using several modules provided with the LASTRAC software.

\section{A. Incompressible Flat-Plate Boundary Layer}

To validate the LASTRAC receptivity module, the receptivity to unsteady suction or blowing in a Blasius boundary layer was computed. Mathematically, this problem is also equivalent to acoustic receptivity due to small amplitude variation in surface admittance. The computed efficiency function for the wall admittance versus Reynolds numbers is shown in Fig. 1. Both parallel (based on linear stability theory) and nonparallel (based on the adjoint PSE approach) results are compared with those obtained from the finite Reynolds number theory [15] in the figure. All three sets of results appear to agree well. It is evident that nonparallel effects are insignificant for this case as the adjoint LST and PSE give almost identical solutions.

\section{B. Incompressible Swept Hiemenz Flow}

The swept Hiemenz flow is an ideal configuration for concept study or code validation for crossflow instability in three-dimensional (3D) boundary layers. The basic flow has a linearly varying streamwise (or equivalent to the chordwise direction in a swept wing boundary layer) and a uniform spanwise velocity component in the free-stream. Bertolotti [14] used this mean flow to validate his nonparallel receptivity calculations using a method that combines the Fourier transform and a Taylor series expansion. A Reynolds number of $\bar{R}=400$ (the Reynolds number based on the spanwise velocity component along the attachment line and a length scale based on the velocity gradient in the chordwise direction at the attachment line) was chosen. Receptivity to localized wall roughness and suction is calculated for four different spanwise wave numbers in Ref.[14]. Figure 2 depicts the efficiency function associated with wall suction for spanwise wave numbers of 0.4 and 0.8 . Results for wall roughness are shown in Fig. 3 for spanwise wave numbers of 0.2 and 0.6. The present results obtained with the adjoint PSE or LST agree well with those from Ref. [14]. It is interesting to note that the adjoint PSE solutions in fact agree with the LNS solutions slightly better than the nonparallel results given in [14]. The results indicate that nonparallel effects are very significant for this mean flow. The difference in efficiency functions between parallel and nonparallel calculations highlights the need to use nonparallel methods such as the adjoint PSE or LNS to accurately predict disturbance amplitudes for crossflow instability near the leading edge. The rapid increase in efficiency function as $x$ is approaching the leading edge (attachment line) is typical for crossflow instability. It is interesting to observe that parallel and nonparallel results are indistinguishable in this region despite their sizable difference further downstream.

It should be noted that since the efficiency function is computed by using the shapefunction evolution along the chordwise direction, transient effects in the PSE marching process would also reflect in the computed receptivity efficiency functions. If receptivity efficiency calculations are of interest in the stable region upstream of the neutral point, a "good" transient free initial shapefunction should be used. This study uses nonparallel local eigensolutions obtained by progressively applying local nonparallel eigenvalue search from the unstable region backward toward the desired upstream location. It was also found that using a shapefunction obtained slightly downstream did not incur strong transient effects; and therefore, the initial location can be pushed further back towards the leading edge. 


\section{Subsonic Flow past Swept Parabolic Cylinder}

Collis and Lele [16] used this geometry to investigate the receptivity of a wall roughness element to stationary crossflow instability. A parabolic cylinder with a leading edge radius of $r_{n}$ (taken to be 1 inch in this study) was chosen for calculations. The sweep angle is 35 degree and the normal Mach number (perpendicular to the leading edge) is 0.8 and the Reynolds number based on the leading edge radius and the normal free stream velocity is $1 \times 10^{5}$. A spanwise periodic roughness element is placed at different chordwise locations to study the receptivity. A Gaussian distribution is used for the chordwise geometry factor of the roughness. The advantage of using the Gaussian shape is that the Fourier transform of the shape is analytically available for comparisons with the available finite Reynolds number based theoretical results. In addition, if the Gaussian bump is sufficiently narrow, the instability modal amplitude at the roughness element location can be computed by simply multiplying the local efficiency function computed by the adjoint PSE calculation and the analytical Fourier geometry factor.

Collis and Lele investigated the receptivity of two spanwise wave numbers $\left(k_{z}=35,100\right.$, where $k_{z}$ is nondimensionalized by $r_{n}$ ). This study compares only the more unstable wave number of 35 . The basic flow was computed by using the thin-layer Navier-Stokes option of the CFL3D code [24] with a grid resolution of 301x161. Figures $4 \mathrm{a}$ and $4 \mathrm{~b}$ compare the computed linear PSE disturbance wave number and growth rate (measured by the integrated total disturbance kinetic energy norm defined in Ref. [1]) with those using LNS from Ref.[16]. The agreement is quite good. The linear PSE solutions were initiated by using a nonparallel eigensolution. Evidently, no noticeable transient effect was present and good agreement with LNS was obtained from the very beginning. The corresponding quasi-parallel LST solutions are also shown in the figure. Substantial nonparallel effect is also apparent for this spanwise wave number. Such nonparallel effects as shown are typical of crossflow instability near the leading edge.

The roughness induced stationary crossflow instability was computed by using the adjoint PSE method developed. For validation purpose, the receptivity was also computed by using the DNS code and linear PSE-based LNS solver mentioned in the previous section. Receptivity efficiency functions for $k_{z}=35$ were computed by the adjoint PSE method at all chordwise locations from very close to the leading edge to a downstream location where significant disturbance growth was attained. The disturbance amplitude evolution is then calculated by using the distributed receptivity integration, Eq. (16). In DNS and LNS, the roughness element is specified in physical space using the Gaussian geometry as described in Ref. [16] with a half-width parameter of $\sigma_{w}=0.01$ (normalized by the nose radius). Figures 5(a) and 5(b) show the disturbance amplitude (normalized by the normal free stream velocity) measured by the peak chordwise velocity perturbation versus the chordwise surface distance (normalized by the nose radius) obtained by all three methods mentioned above for roughness elements located at two different chordwise locations. The spikes in the DNS and LNS solutions indicate the excitation of a broad range of waves near the roughness source. It should be mentioned that the PSE-based LNS calculation was performed with a constant chordwise wave number set at the instability eigenmode value right at the source location to relieve the streamwise grid resolution required. As a result, noticeable differences with DNS can be observed near the roughness source. However, the LNS solution quickly merges into the eigenmode not too far downstream where all three solutions begin to agree well among one another. These results suggest that even though continuous spectrum and multiple modes are present near the source, the contribution from the eigenmode of interest is the most, if not the only, important part that needs to be resolved in this case. The adjoint PSE solution does not contain any continuous spectrum or other eigenmode near the source, thus no spike is present in the amplitude evolution curve.

The good agreement after the eigenmode has developed downstream of the source also indicates that the effective amplitude at the source location evaluated by extrapolation based on the linear PSE N-factor prediction using

$$
A_{\text {eff }}(x)=A(x) / \exp \left(N(x)-N_{0}\right)
$$

would agree among all three methods. In the above expression, linear PSE $\mathrm{N}$-factor at the source location is indicated by $N_{0}$. The adjoint PSE amplitude evolution shown in the figure is computed by assuming a finite size roughness element. When the nonuniformity source is compact, one can compute the local disturbance amplitude by 
simply multiplying the geometry factor with the local receptivity efficiency function. This study repeats the DNS and LNS calculations for an element width of 0.01 at several chordwise locations. Figure 6 compares the results with adjoint LST and PSE predictions obtained by assuming compact sources locally at each chordwise location. The agreement with DNS and with PSE-based LNS for sources located at $x \geq 2$ is quite good. On the other hand, the quasi-parallel adjoint LST solution significantly overpredicts the disturbance amplitudes. This demonstrates the importance of using a nonparallel prediction method for such flow configurations. More calculations are necessary to clarify the PSE-based LNS accuracy at smaller $x$.

To assess the source compactness assumption, the adjoint PSE calculations were repeated for several roughness widths at $x=1$. The resulting amplitudes are shown in Fig. 7 for $\sigma_{w}$, ranging from 0.01 to 0.2 . Wider roughness elements are more receptive to stationary crossflow disturbances. The generated amplitude from a half-width of 0.2 is more than an order of magnitude larger than that from 0.01 . The equivalent amplitudes calculated by using Eq. (21) show that the compact source assumption is valid for a half-width less than 0.1 . All the results presented for this test case show that receptivity computed by using the adjoint PSE method yield accurate amplitude predictions, comparable to a higher fidelity DNS or LNS calculation. In the following subsection, the adjoint PSE method is used for receptivity studies of two of the available swept wing experiments.

\section{Incompressible ASU Swept Wing Boundary Layer}

Janke [17] investigated the incompressible ASU swept wing experiment [19] from the perspective of receptivity and the SPRE based control of stationary crossflow disturbances. Finite Reynolds number theory and LNS were used in Ref. [17] for receptivity calculations. The incompressible ASU swept wing experiment was performed on a swept wing geometry based on the NLF (2)-0415 airfoil with an angle-of-attack of -4 degrees and a sweep angle of 45 degrees. Janke [17] changed the angle of attack to -3.5 degree in order to better match the experimental $\mathrm{Cp}$ distribution. This study recomputed the inviscid $\mathrm{Cp}$ distribution by accounting for the wind tunnel wall blockage effect using CFL3D [24] for the installed angle-of-attack of -4 degrees. Figure 8 compares the calculated Cp distribution with that from the experimental data and Janke [17]. It appears that the calculated $\mathrm{Cp}$ distribution does not match with experimental data as well as Ref. [17]. To assess the effect of different mean flow on the stability results, the computed linear PSE N-factor using the current mean flow is compared with that by Janke for a spanwise wavelength of $12 \mathrm{~mm}$ in Fig. 9. The agreement is good for $x / c$ up to about 0.4 . This seems to be in line with the difference in $\mathrm{Cp}$ distribution for which the slope is quite close for $x / c$ from 0.1 to about 0.4 . However, there exists substantial difference in $\mathrm{Cp}_{\mathrm{p}}$ for $x / c<0.1$.

Despite some agreement in the linear PSE results, the computed roughness efficiency functions shown in Fig. 10 exhibit substantial differences. The difference in the mean flows in fact has more impact on the receptivity results. Exploring the effects of different $\mathrm{Cp}$ distributions on receptivity and stability results is beyond the scope of this paper. More studies are necessary to clarify the mean flow discrepancies and assess how well the current adjoint PSE-based receptivity approach predicts the disturbance amplitude for a given roughness configuration.

\section{E. Supersonic Swept wing Boundary Layers}

The ASU LFC experiments reported in [3] were conducted in the Mach 2.4 ASU 0.2-m supersonic wind tunnel. The swept wing model had a leading edge sweep of 73 degrees, a 0.3 meter streamwise chord at the mid-span location and a symmetric cross section with thickness-to-chord ratio of $4 \%$. The free stream temperature and Mach number are 138 degree $\mathrm{K}$ and 2.4, respectively. Successful SPRE passive control was demonstrated for this configuration at various chord Reynolds numbers from 8 through 16.3 million [3]. This swept wing model was previously investigated computationally in refs. [4, 27]. The highest Reynolds number case was chosen for the computations reported here.

Receptivity calculations were performed for a stationary crossflow mode with a spanwise wavelength of $3 \mathrm{~mm}$. Figure 11 shows the computed roughness-induced efficiency functions for both parallel (adjoint LST) and nonparallel (adjoint PSE) calculations. Although not compared in the figure, the adjoint LST results agree very well with previous calculations using an alternate numerical implementation of the finite Reynolds number theory [4]. It is also evident that the nomparallel results are close to those based on the parallel theories for $\mathrm{x} / \mathrm{c}>2 \%$. Overall, 
receptivity in the leading edge region becomes less efficient after accounting for flow nonparallelism. This trend is consistent with previous results for incompressible boundary layers [14, 17]. The magnitude of nonparallel effects in this case is considerably less than the incompressible ASU swept wing [17], or the swept Hiemenz flow [14] cases. It may be a direct outcome of the larger Reynolds number in this supersonic configuration. Further studies for a moderate Reynolds number may help clarify this issue.

Before performing receptivity calculations, we first investigate the Fourier geometry factors of the roughness array of interest. It has been shown in our previous work [4] that the magnitude of the geometry factor varies significantly with the radius of the cylindrical roughness elements and there exists an "optimal" radius that would lead to a larger geometry factor; and in turn, more efficient excitation. We model the controlled excitation as an array of roughness elements with a cylindrical planform. For a roughness array located at $x / c=0.04$ with a height of 6 microns and a spanwise spacing of $1.7 \mathrm{~mm}$, we perform a discrete Fourier transform for various dot diameters. The computed chordwise distribution $\widetilde{F}(x, \beta)$ is then used to compute the geometry factor for the dot diameter given using the following Fourier integral:

$$
\widetilde{F}_{w}=\int_{\alpha_{x}} \widetilde{F}(\xi, \beta) \exp \left(-i \theta_{r}(\xi, \beta)\right) d \xi
$$

where the exponent is defined in Eq.(17) and the chordwise wave number is from the computed linear PSE results. The integration range covers the whole roughness element. Note that this geometry factor integral excludes the effect of the receptivity efficiency function, as can be seen by comparing with Eq. (16). It was created artificially as a quantity to measure the integral effects of the instability wave and the geometry factor given. Figure 12 depicts the computed geometry factor for a diameter ranging from 100 to 1200 microns. Also shown in the figure is the disturbance amplitudes predicted at a slightly downstream location $(x / c=0.045)$. Similar to the results shown in Ref.[4], the results indicate a double hump variation and it appears to have maxima around 350 and 800 microns. However, surprisingly, the disturbance amplitude generated remains monotonically increasing with the roughness diameter. The decrease of the roughness geometry factor between 400 and 600 does not translate into a less efficient disturbance generation process, although, there is a change of slope in the amplitude variation in this region to reflect the geometry factor variation. To view this difference more clearly, we plot in Fig. 13 the amplitude evolution predicted by integrating the receptivity and linear amplification simultaneously using Eqs. (13) and (16) for three dot diameters. All disturbance amplitudes presented herein refer to the peak chordwise velocity amplitudes normalized by the total free stream velocity of $565 \mathrm{~m} / \mathrm{s}$. It clearly shows that despite the decrease in geometry factor when the diameter is increased from 400 to 600 , the overall disturbance amplitude remains the increasing trend for a larger diameter. This outcome is consistent with earlier work that a spatial integral that accounts for variations in efficiency function and instability wavenumber across the nonuniformity must be used for a finite-size surface nonuniformity [26]. This is particularly true for nonuniformity distributions that lead to an oscillatory behavior of geometry factor as function of the element size. Non-parallel effects reduce or eliminate the destructive interference between instability wave contributions from different parts of the nonuniformity and, therefore, the minima predicted by purely localized theory are not observed as seen from Fig. 13.

Another factor that could strongly influence the disturbance amplitude generated by roughness elements is the chordwise location. For a diameter of 500 microns and a height of 6 microns, the array location was varied from $x / c$ $=0.01$ to 0.05 , and the resulting amplitudes measured at $x / c=0.08$ (a location where a complete eigenmode would have been formed) are summarized in the following table:

\begin{tabular}{|c|c|}
\hline Roughness location $(x / c)$ & $\mathrm{A}($ at $x / c=0.08)$ \\
\hline 0.01 & 0.3183 \\
\hline 0.02 & 0.0600 \\
\hline 0.03 & 0.0244 \\
\hline 0.04 & 0.0108 \\
\hline 0.05 & 0.0049 \\
\hline
\end{tabular}

Larger amplitude at a downstream location implies more effective disturbance generation at the array location. The disturbance amplitude generated appears to be commensurate with the receptivity efficiency function variation near 
the leading edge. The most effective location seems to be near the attachment line. More calculations with better resolved mean flow near the attachment line are necessary to see if the effective amplitude decrease again very close to the leading edge as was observed in both Ref. [16] and Fig. 6 for the subsonic swept cylinder case.

To simulate the SPRE control of the stationary crossflow instability, this study performed a series of nonlinear PSE calculations with initial amplitudes predicted by the adjoint PSE approach. The most amplified stationary disturbance appears to have a spanwise wavelength of about $3 \mathrm{~mm}$. It is assumed that the control roughness elements array is located at $x / c=0.02$ with a spacing of $1.7 \mathrm{~mm}$, a diameter of 500 microns, and a cylindrical height of 6 microns. To include the effect of the control input on the most amplified $3 \mathrm{~mm}$ mode by using a small number of terms kept in the Fourier series, the geometry factors of $1.5,0.75$, and $0.5 \mathrm{~mm}$ for the given roughness array were computed. Using these computed geometry factors in conjunction with the corresponding efficiency functions, this study obtains the disturbance amplitude variation shown in Fig. 14. Among all three harmonics shown, only the 1.5 and $0.75 \mathrm{~mm}$ modes appear to have significantly large amplitudes to affect the most unstable crossflow mode. The amplitudes at $x / c=0.027$ are $0.33 \%, 0.13 \%$, and $0.011 \%$ for the $1.5,0.75$, and $0.5 \mathrm{~mm}$ mode, respectively. To estimate the amplitude of the $3 \mathrm{~mm}$ mode, a geometry factor artificially generated at each chordwise direction by multiplying the maximum roughness height of 0.1 microns with a random number between 0 and 1 was used. The amplitude integration was then performed using the efficiency function for a spanwise wave length of $3 \mathrm{~mm}$. By varying the seeding of the random number generator, various calculations were performed. The amplitudes of the 3 $\mathrm{mm}$ mode at $x / c=0.027$ were found to vary in the range of $1-3 \times 10^{-4}$. A value of $3 \times 10^{-4}$ was chosen as a conservative estimate. Similarly, the naturally excited amplitudes of the $1.5,0.75$, and $0.5 \mathrm{~mm}$ modes were estimated to be $5 \times 10^{-4}, 6 \times 10^{-4}$, and $1 \times 10^{-8}$, respectively. It should be noted that more reasonable estimates may be obtained by employing a more rigorous stochastic model for the polished wing surface.

Nonlinear PSE calculations were performed by using a fundamental spanwise wavelength of $3 \mathrm{~mm}$. Four modes, $(0$, $1),(0,2),(0,3)$, and $(0,4)$ were initiated by imposing the amplitudes predicted above and higher harmonics were generated via nonlinear interaction. The disturbance phase difference among the excited 1.5, 0.75, and $0.5 \mathrm{~mm}$ modes computed by the receptivity calculation (using Eqs. (13) and (16)) is retained in the nonlinear PSE calculations. To simulate the uncontrolled case, the amplitudes of the first four harmonics were obtained by a random geometry factor as described above. The resulting disturbance evolution is shown in Fig. 15 for both controlled and uncontrolled cases. The presence of the roughness array clearly delays the rapid growth and saturation of the most amplified $3 \mathrm{~mm}$ mode. The estimates for the initial amplitudes of the $(0,2)$ and other higher modes may not reflect the true amplitude for naturally excited disturbances. If these initial amplitudes were lowered to $1 \times 10^{-8}$, the uncontrolled disturbance evolution became that shown in Fig. 16. The rapid growth änd saturation location moves further upstream as compared to that shown in Fig. 15. This implies that more energetic harmonic modes, as a matter of fact, help delay the spurt of the $3 \mathrm{~mm}$ mode. A more reliable way to predict receptivity of naturally excited disturbances is necessary to have a more realistic prediction for the uncontrolled case. Both results indicate that reduced growth of the most amplified stationary crossflow mode can be achieved by the presence of the periodic array of roughness elements used in the ASU experiments.

It was noted in the experiment that the nominal roughness diameter was designed to be 400 microns even though the real size was estimated to be 500 microns. To assess the effect of the size of roughness elements, the controlled case was repeated by using a roughness diameter of 400 and 600 microns. Figure 17 compares the modal amplitude variations for the most unstable $(3 \mathrm{~mm})$ and control $(1.5 \mathrm{~mm})$ modes for all three roughness diameters. The same initial amplitude of $3 \times 10^{-4}$ was used for the $3 \mathrm{~mm}$ mode for all three calculations. A larger diameter causes the control mode to grow earlier near the leading edge. However, the control mode peaks at about the same level near the $15 \%$ chord for all three element sizes. The small difference in the control mode evolution near the leading edge eventually leads to substantial differences in the location of rapid rise and saturation level of the modal amplitude of the $3 \mathrm{~mm}$ mode further downstream. A larger roughness element diameter appears to move the location of rapid rise farther downstream but with a higher saturation amplitude. Without detailed analysis using the secondary instability theory (e.g. Ref.[25]), it is premature to judge the merit of a larger diameter in terms of transition location. A more comprehensive parametric study is necessary in order to determine whether an optimal roughness size indeed exists.

The above calculations demonstrate that an integrated transition prediction procedure capable of accounting for the disturbance environment may be performed from the receptivity stage all the way up to highly nonlinear stage. For 
stationary crossflow instability dominated configurations, further secondary instability analysis as described in[25] should be performed to determine the estimated transition location.

\section{Concluding Remarks}

A localized receptivity method based on the adjoint PSE approach for compressible stability equations has been developed. Receptivity efficiency functions are calculated locally for wall suction or roughness elements using a numerically efficient marching procedure. The calculated receptivity efficiency function provides the Green's function to the disturbance amplitude evolution in a nonparallel (growing) boundary layer. Given the geometry distribution along the chordwise direction, the linear disturbance amplitude evolution for a finite size, distributed nonuniformity can be computed by evaluating the integral effects of both disturbance generation and linear amplification. In contrast to other prediction methods in physical space such as the DNS or LNS, these tabulated efficiency functions may be repeatedly used to predict disturbance amplitude for any given surface nonuniformity configurations.

This study has validated the theoretical and computational approach by comparing present results with those from DNS and LNS procedures. For several test configurations, the generated disturbance amplitudes computed by the adjoint PSE method agree well with LNS and DNS results. Therefore, for highly nonparallel cases, such as the receptivity near the leading edge of a swept wing boundary layer, the adjoint PSE method should provide an efficient yet accurate alternative to the time-consuming LNS or DNS methods for both incompressible and compressible boundary layers. The adjoint PSE and the reduced LST methods have been implemented as a new module in the physics based advanced transition prediction tool LASTRAC.

For the supersonic swept wing test configuration, the study used LASTRAC to demonstrate how to incorporate the new receptivity module in conjunction with the nonlinear PSE module to perform an integrated transition prediction. This procedure begins from the geometry of an array of periodic roughness elements, and ends with nonlinear disturbance amplitudes saturation that would eventually leads to secondary instability and transition. The absolute disturbance amplitude is predicted without resorting to adjustment in the disturbance level. Of course, further work is necessary to handle the naturally excited disturbances. The present research serves as a starting point to incorporate the disturbance environment information in a physics-based, high fidelity integrated transition prediction methodology for advanced concept studies in future aerospace vehicle design.

\section{Acknowledgement}

The authors would like to thank Dr. Li Jiang from the University of Texas at Arlington for kindly providing the mean flow and DNS calculations for the parabolic cylinder. Thanks are also due to Drs. Janke and Balakumar for technical communication related to Ref. [17].

\section{References}

1. Chang, C.-L., "The Langley Stability and Transition Analysis Code (LASTRAC): LST, Linear and Nonlinear PSE for 2-D, Axisymmetric, and Infinite Swept Wing Boundary Layers," AIAA Paper 20030974, 2003.

2. Chang, C.-L., "LASTRAC.3d: Transition Prediction in 3D Boundary Layers," AIAA Paper 2004-2542. Portland, Oregon, Jun. 2004.

3. Saric, W. S. and Reed, H. L., "Supersonic Laminar Flow Control on Swept Wings Using Distributed Roughness," AIAA Paper 2002-0147, 2002.

4. Choudhari, M., Chang, C.-L., Streett, C. L., and Balakumar, P., "Integrated Transition Prediction: A Case Study in Supersonic Laminar Flow Control," AIAA Paper 2003-0973, 2003.

5. Choudhari, M. and Streett, C. L., "Boundary Layer Receptivity Phenomena in Three-Dimensional and High-Speed Boundary Layers," AIAA Paper 1990-5258, 1990.

6. Choudhari, M. and Streett, C. L., "Theoretical Prediction of Boundary-Layer Receptivity," AIAA Paper 1994-2223, 1994.

7. Crouch, J. D., "Receptivity of Boundary Layers," AIAA Paper 1994-2224, 1994.

12

American Institute of Aeronautics and Astronautics 
8. Goldstein, M. E., "Scattering of Acoustic Waves into Tollmien-Schlichting Waves by Samll Streamwise Variations in Surface Geometry, "J. Fluid Mech., Vol. 154, pp. 509-529, 1985.

9. Ruban, A. I., "On the Generation of Tollmien0Schilichting Waves by Sound," Fluid Dyn., Vol. 19, pp.709$716,1985$.

10. Hill, D. C., "Adjoint Systems and Their Role in the Receptivity Problem for Boundary Layers," J. Fluid Mech., Vol. 292, pp. 183-204, 1995.

11. Herbert, Th., "Parabolized Stability Equations," Annu. Rev. Fluid Mech., Vol. 29, p. 245, 1997.

12. Airiau, C., Walther, S., and Bottaro, A., "Nonparallel Receptivity and the Adjoint PSE," in Proc. of the $5^{\text {th }}$ IUTAM Symposium on Laminar-Turbulent Transition (Springer-Verlag, Berlin, 1999), pp.57-62.

13. Collis, S. and Dobrinsky, A., "Evaluation of Adjoint Based Methods for the Prediction of Linear and Nonlinear Receptivity," in Proc. of the $5^{\text {th }}$ IUTAM Symposium on Laminar-Turbulent Transition (SpringerVerlag, Berlin, 1999), pp. 111-116.

14. Bertolotti, F. P., "Receptivity of Three-Dimensional Boundary-Layers to Localized Wall Roughness and Suction," Phys. Fluids, Vol. 12, No. 7, pp. 1799-1809, 2000.

15. Choudhari, M. and Streett, C. L., "A Finite Reynolds-Number Approach for the Prediction of BoundaryLayer Receptivity in Localized Regions," Phys. Fluids A, Vol. 4, No. 11, pp.2495-2514, 1992.

16. Collis, S. S. and Lele, S. K., "Receptivity to Surface Roughness near a Swept Leading Edge," J. Fluid Mech. Vol. 380, pp. 141-168, 1999.

17. Janke, E., "Receptivity and Transition Control of Swept wing Boundary-Layers; Effects of Surface Curvature and Nonlinearity," AIAA Paper 2001-2980, 2001.

18. Tumin, A. M. and Fedorov, A. V., "Instability Wave Excitation by a Localized Vibrator in the Boundary Layer," J. Appl. Mech. Tech. Phys., Vol. 25, p. 867, 1984.

19. Saric, W. S., Carillo, R. B., and Reibert, M. S., "Leading Edge Roughness as a Transition Control Mechanism," AIAA Paper 98-0781, Jan. 1998.

20. Jiang, L., Choudhari, M., Chang, C.-L., and Liu, C., "Direct Numerical Simulations of Instability-Wave Generation and Propagation in Supersonic Boundary Layers," Lecture Notes in Computer Science, vol. 2668, pp. 859-870, 2003.

21. Jiang, L., Choudhari, M., Chang, C.-L., and Liu, C., "Direct Numerical Simulations of Crossflow Disturbances in Supersonic Boundary Layers," AIAA Paper 2004-589, 2004.

22. Streett, C. L., "Direct Harmonic Navier-Stokes Methods for Efficient Simulation of Weakly-Nonlinear Wave Packets," AIAA Paper 1998-0784, 1998.

23. Guo, Y., Malik, M. R., and Chang, C.-L., "A Solution Adaptive Approach for Computation of Linear Waves," AIAA Paper 1997-2072, 1997.

24. http://cfl3d.larc.nasa.gov/Cfl3dv6/cfl3dv6.html [cited 5 Jan. 2005].

25. Malik, M. R., Li, F., Choudhari, M. M., and Chang, C.-L., "Secondary Instability of Crossflow Vortices and Swept wing Boundary-Layer Transition," J. Fluid Mech., vol. 399, pp. 85-1 15, 1999.

26. Choudhari, M., "Distributed acoustic receptivity in laminar flow control configurations," Phys. Fluids, Vol. 6, Feb, pp. 489-506, 1994.

27. Choudhari, M. M., Chang, C.-L. and Jiang, L., "Towards Transition Modeling for Supersonic Laminar Flow Control," Philosophical Transactions of the Royal Society Series A: Mathematical, Physical and Engineering Sciences, to appear, 2005. 


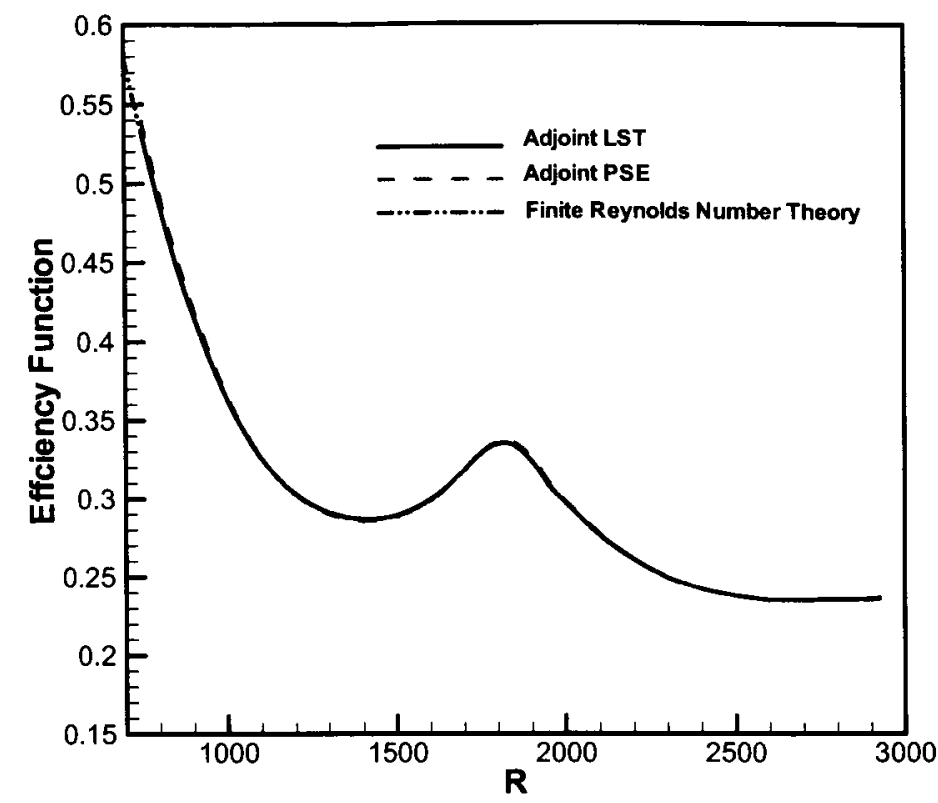

Figure 1: Computed efficiency function magnitude for wall suction induced receptivity versus Reynolds number for a 2D disturbance with frequency of $F=0.35 \times 10^{-6}$ in a Blasius boundary layer.

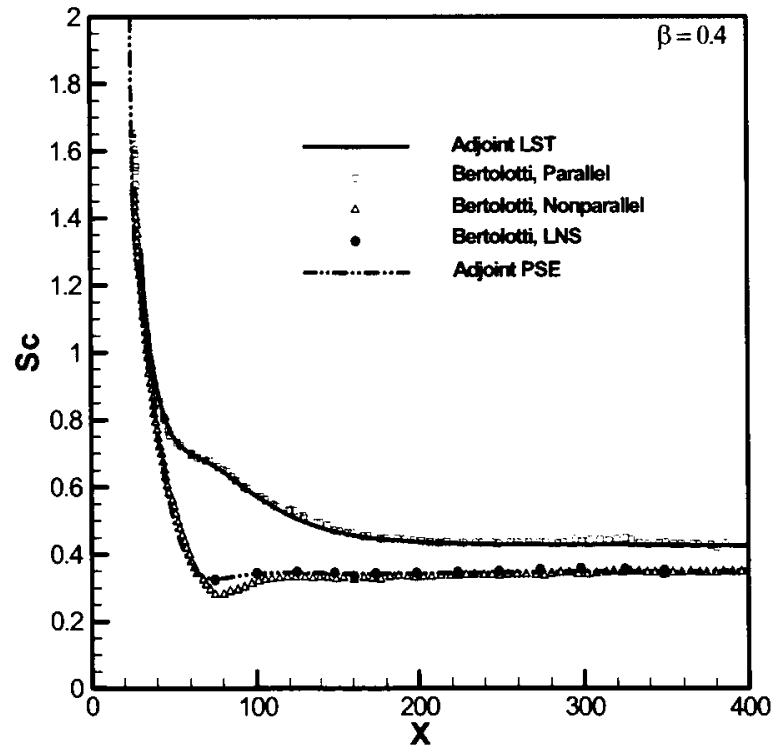

(a)

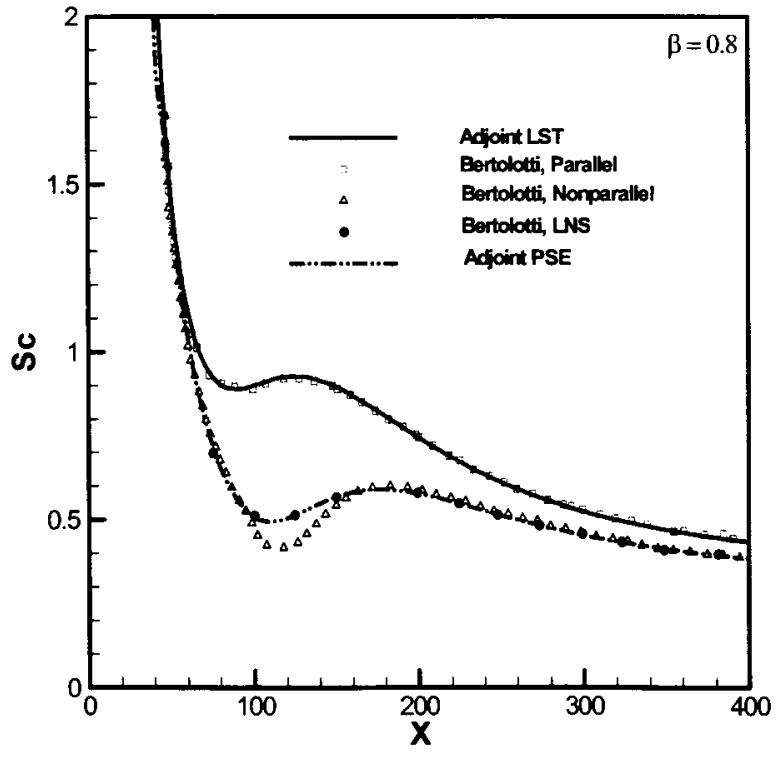

(b)

Figure 2: Comparison of receptivity efficiency function due to wall suction with parallel, nonparallel, and linear Navier-Stokes results from Bertolotti $\lceil 3\rceil$ for swept Hiemenz flow: (a) $\beta=0.4$ and (b) $\beta=0.8$. 


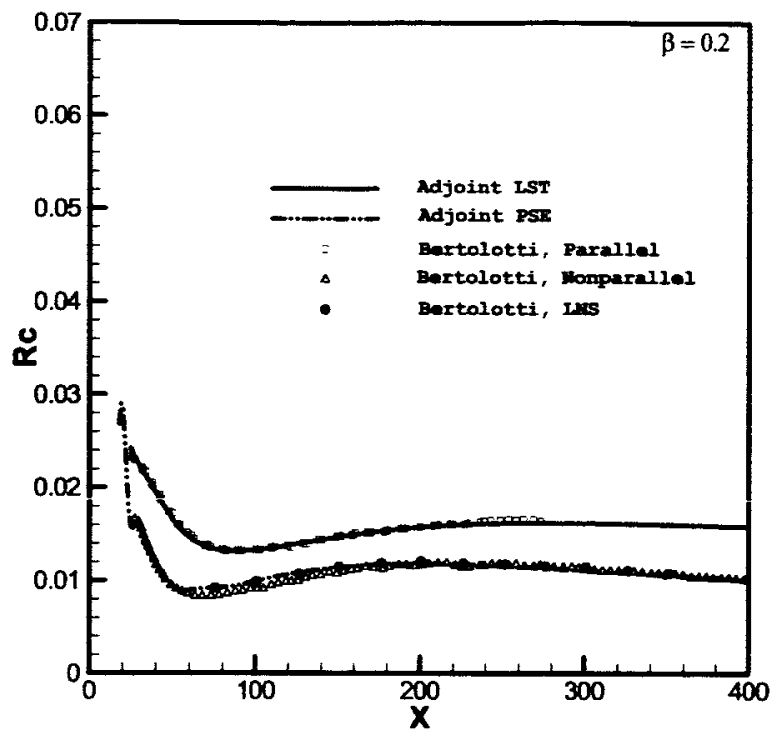

(a)

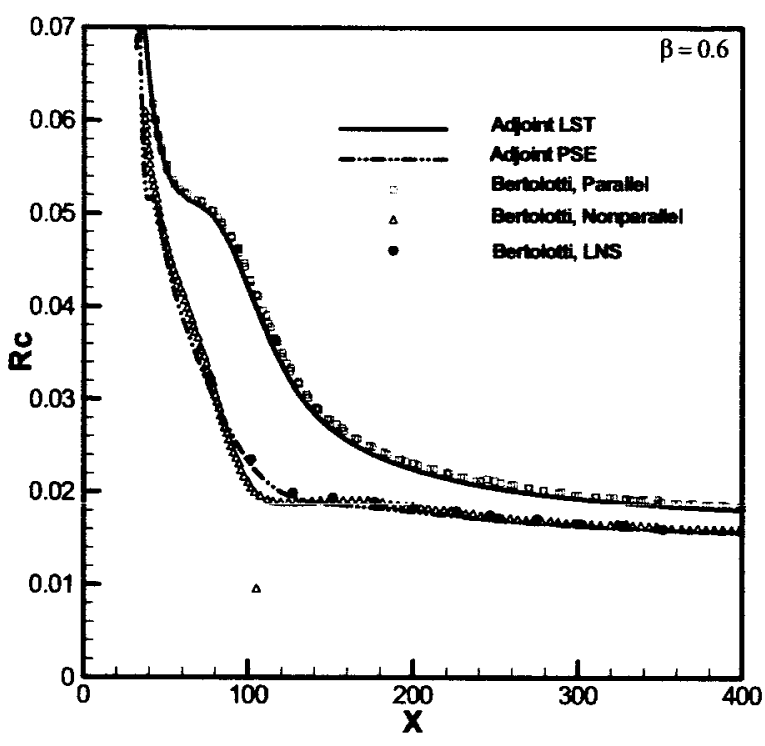

(b)

Figure 3: Comparison of receptivity efficiency function due to wall roughness with parallel, nonparallel, and linear Navier-Stokes results from Bertolotti[3] for swept Hiemenz flow: (a) $\beta=0.2$ and (b) $\beta=0.6$.

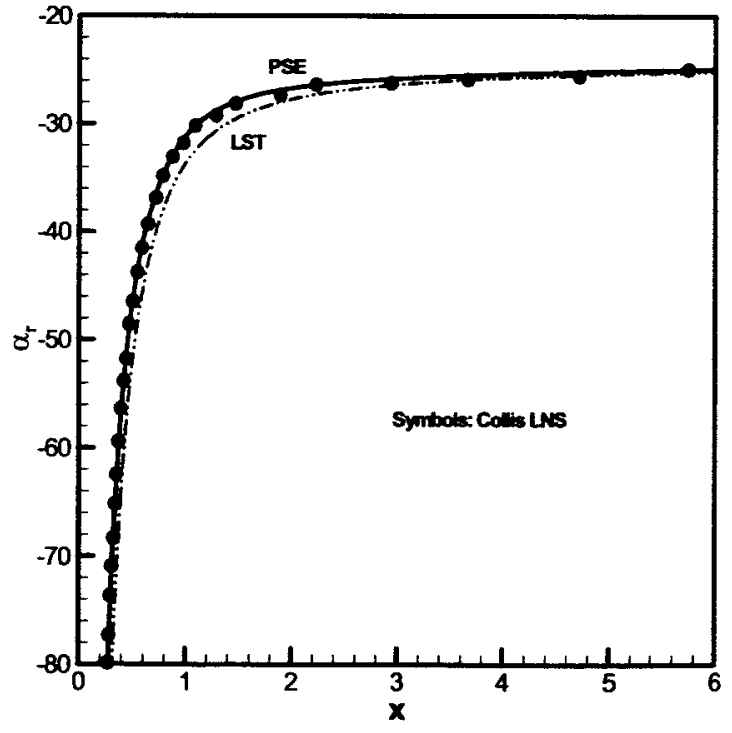

(a)

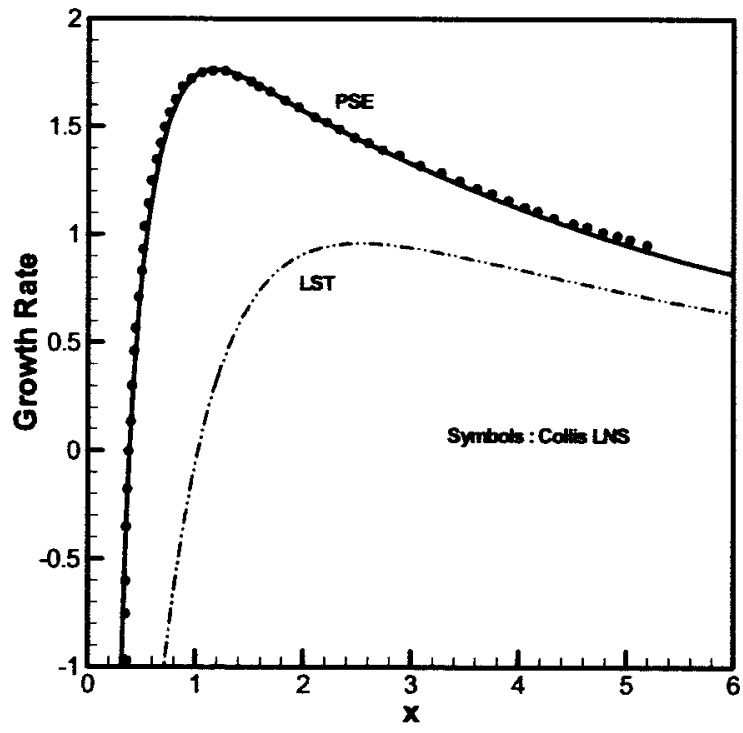

(b)

Figure 4: Comparison of computed chordwise (a) wave number and (b) growth rate with LNS solutions from Collis and Lele [16] for incompressible parabolic cylinder $\left(k_{z}=35\right)$. 


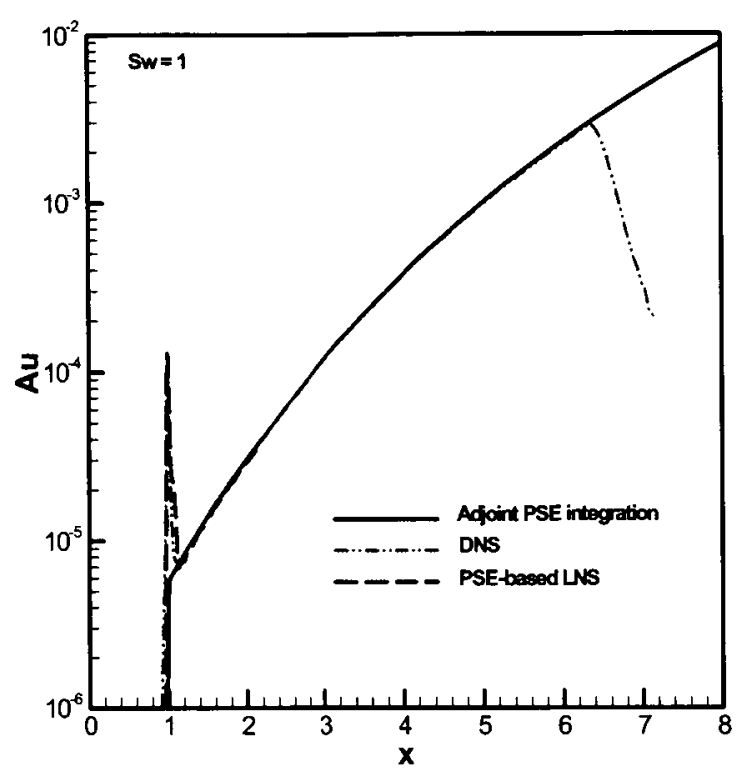

(a)

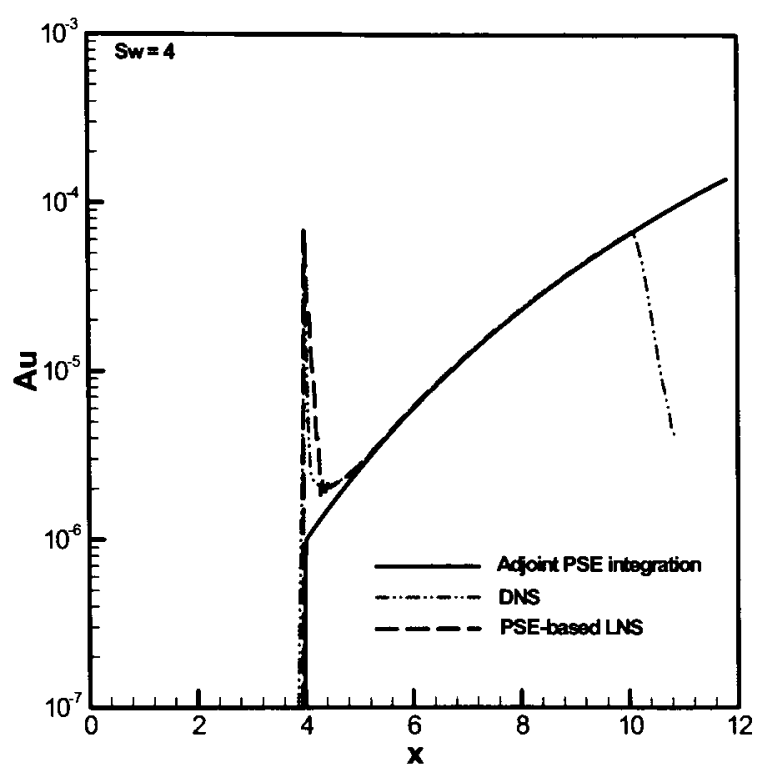

(b)

Figure 5: Disturbance amplitude versus chordwise distance for a roughness element located at (a) $x=1$ (b) $x=$ 4 (results computed by using adjoint PSE, DNS, and PSE-based LNS) for incompressible parabolic cylinder $\left(k_{z}=35\right)$.

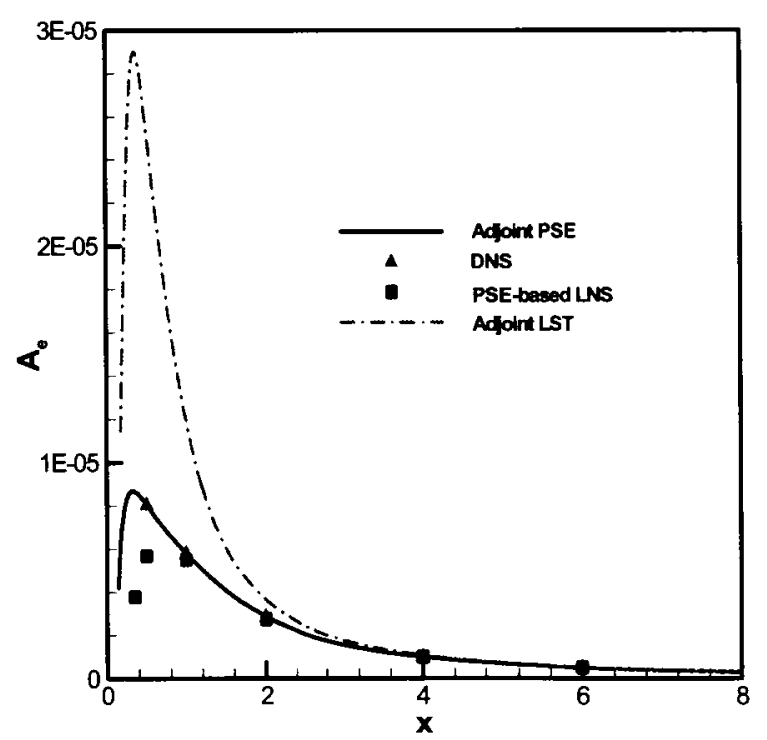

Figure 6: Disturbance amplitude in response to a local roughness element computed by adjoint PSE, adjoint LST, DNS, and LNS for parabolic cylinder.

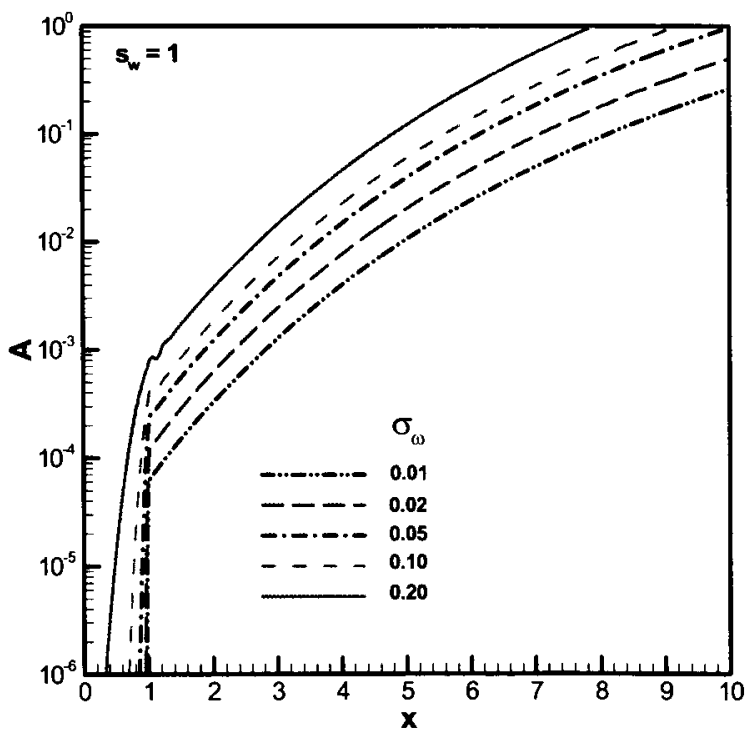

Figure 7: Disturbance amplitude versus chordwise distance for roughness elements with various widths for parabolic cylinder. 


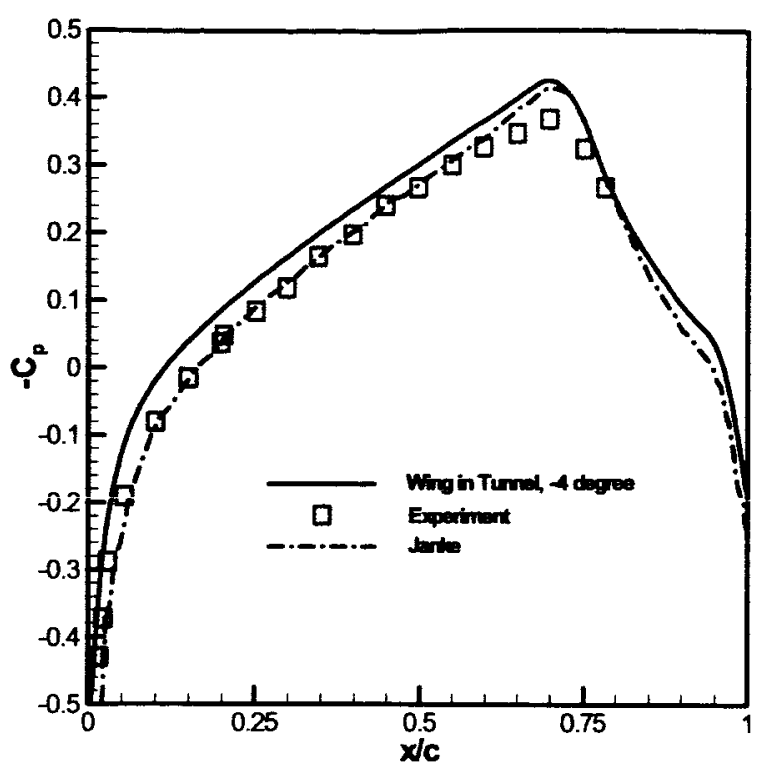

Figure 8: Cp distribution for the incompressible ASU swept wing experiment; comparing with experimental data and Janke[17].

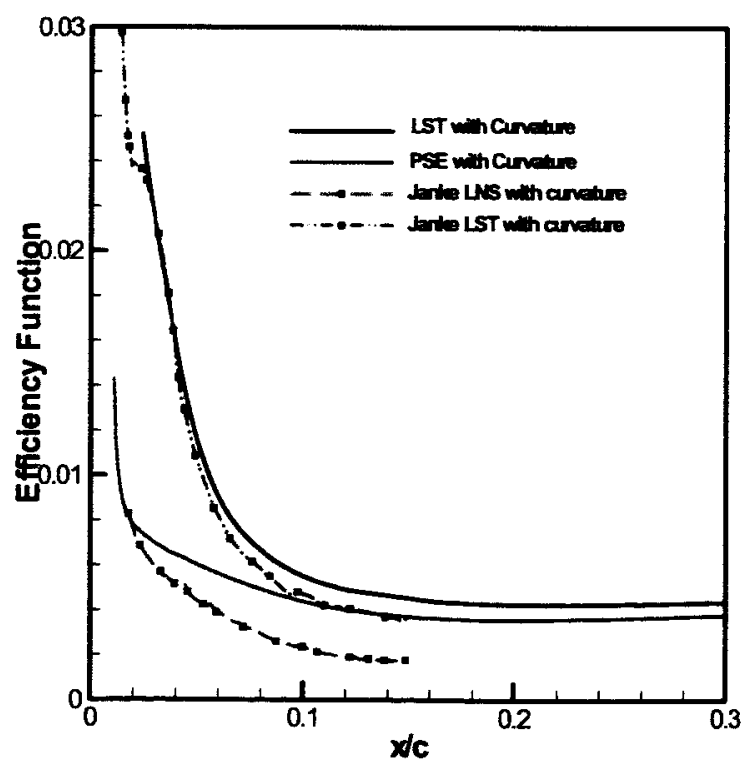

Figure 10: Comparison of computed receptivity efficiency function due to wall roughness for a spanwise wavelength of $12 \mathrm{~mm}$ using adjoint LST and PSE with theoretical and LNS results of Janke[17].

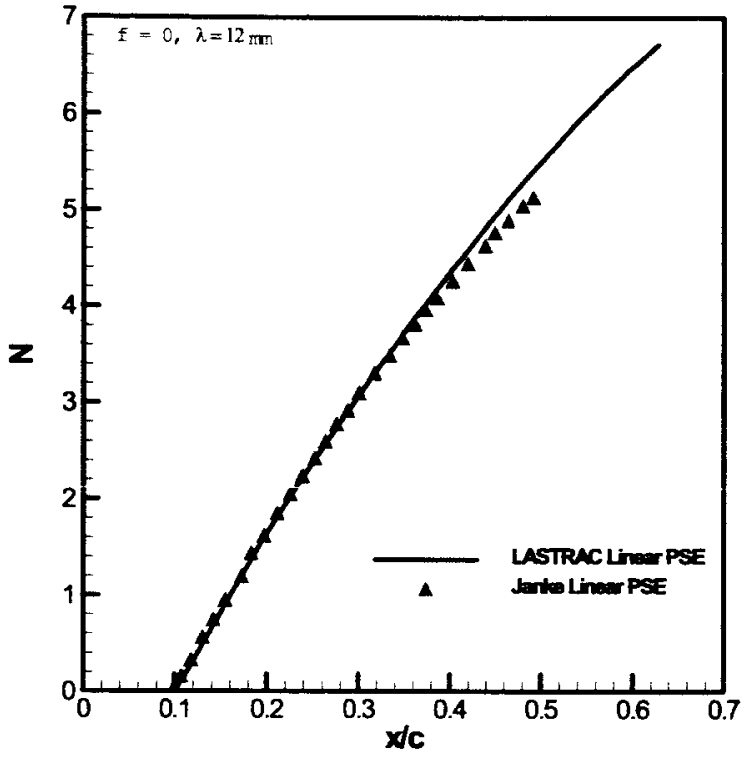

Figure 9: Comparison of the computed linear PSE Nfactor for stationary crossflow (with a spanwise wavelength of $12 \mathrm{~mm}$ ) with Janke[17].

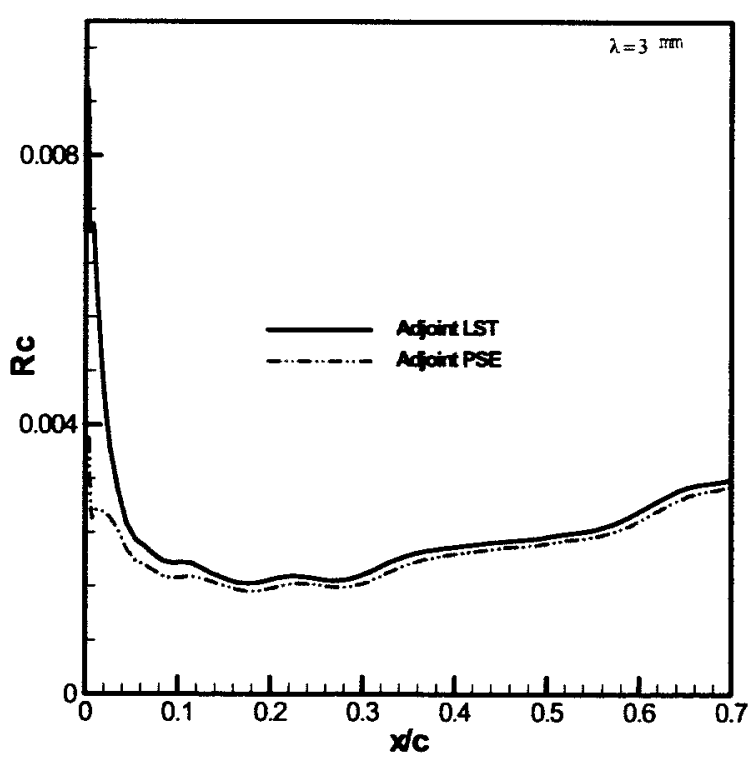

Figure 11: Receptivity efficiency functions due to wall roughness computed by adjoint LST and PSE for the Mach 2.4 ASU experiment with a spanwise wavelength of $3 \mathrm{~mm}$. 


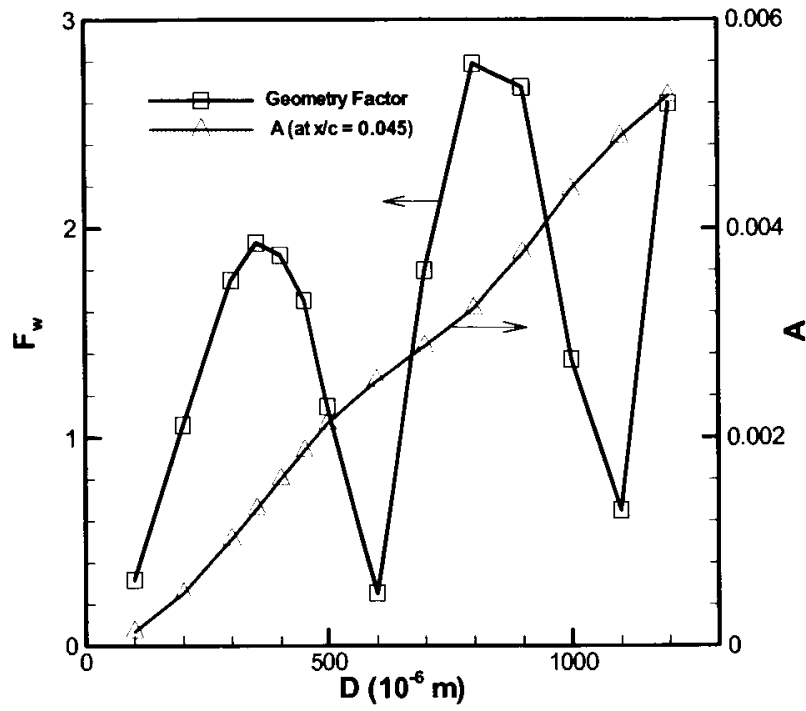

Figure 12: Geometry factor of a periodic array of roughness elements located at $x / c=0.04$, obtained by Fourier transform in the chordwise and spanwise directions. Disturbance amplitudes behind the array are also shown.

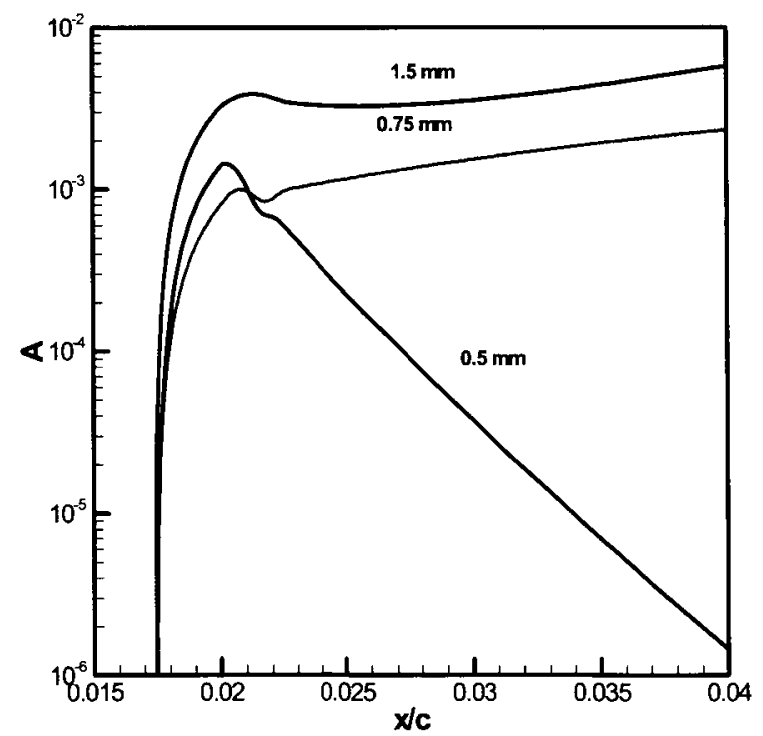

Figure 14: Disturbance amplitude computed by adjoint PSE for an array of roughness elements located at $x / c=$ 0.02 : showing first 3 harmonics of the fundamental wave length of $1.5 \mathrm{~mm}$.

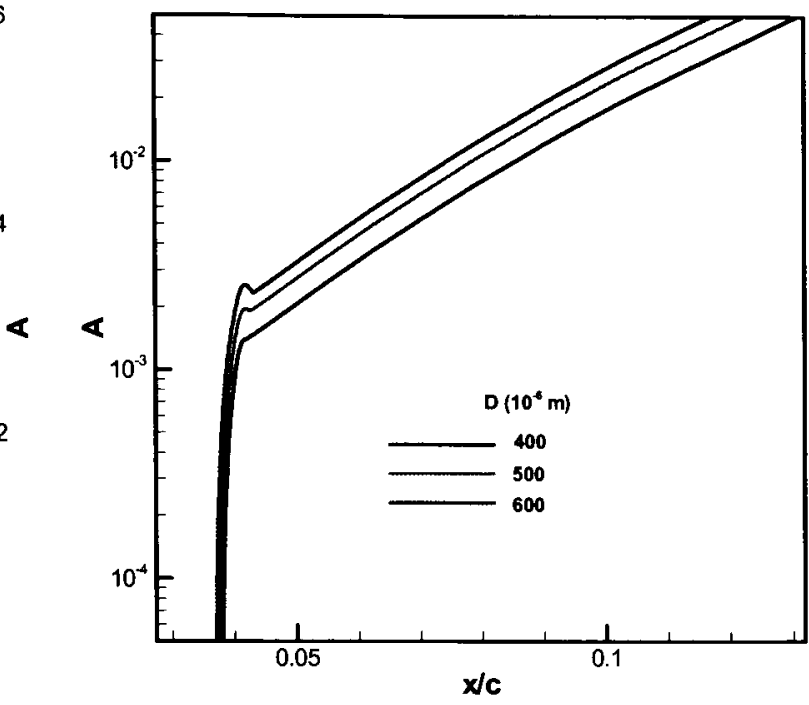

Figure 13: Disturbance amplitudes variation for a roughness array located at $x / c=0.04$ with various dot diameters.

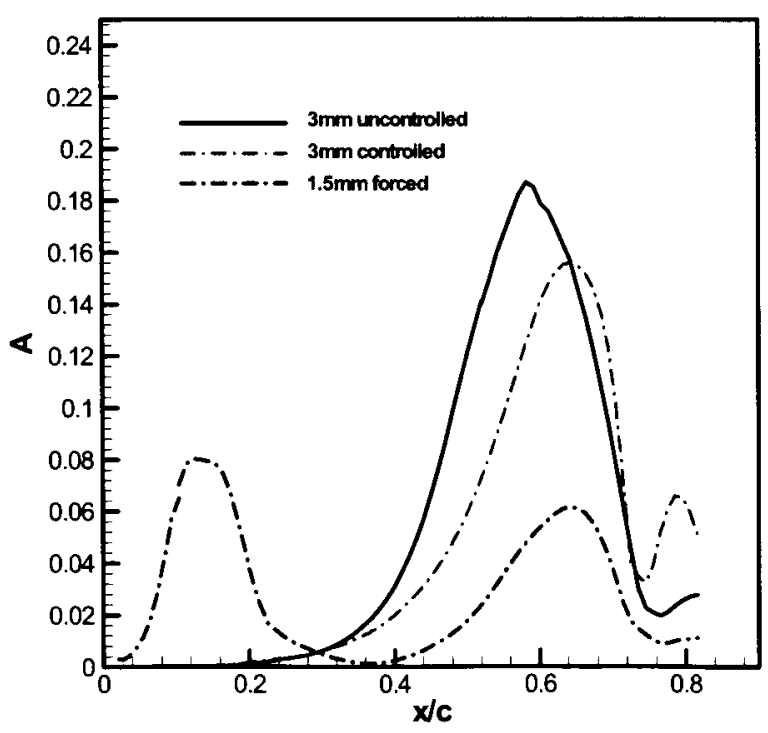

Figure 15: Nonlinear amplitude variation of the $3 \mathrm{~mm}$ mode with and without the input of the controlled modes from the results shown in Fig. 16. 


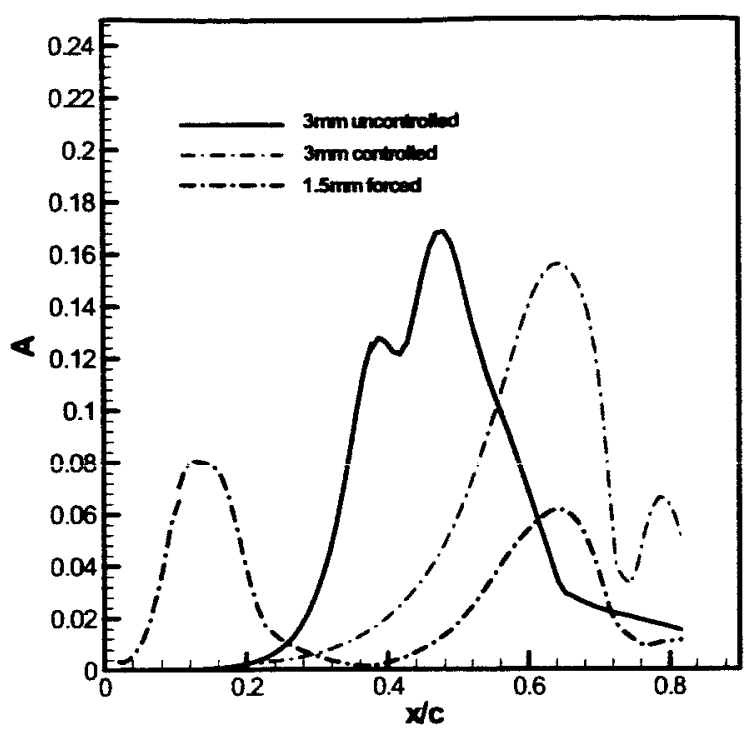

Figure 16: Nonlinear amplitude variation of the $3 \mathrm{~mm}$ mode with and without the input of the controlled inputs from the results shown in Fig. 15 ; assuming a small initial amplitude for the harmonics.

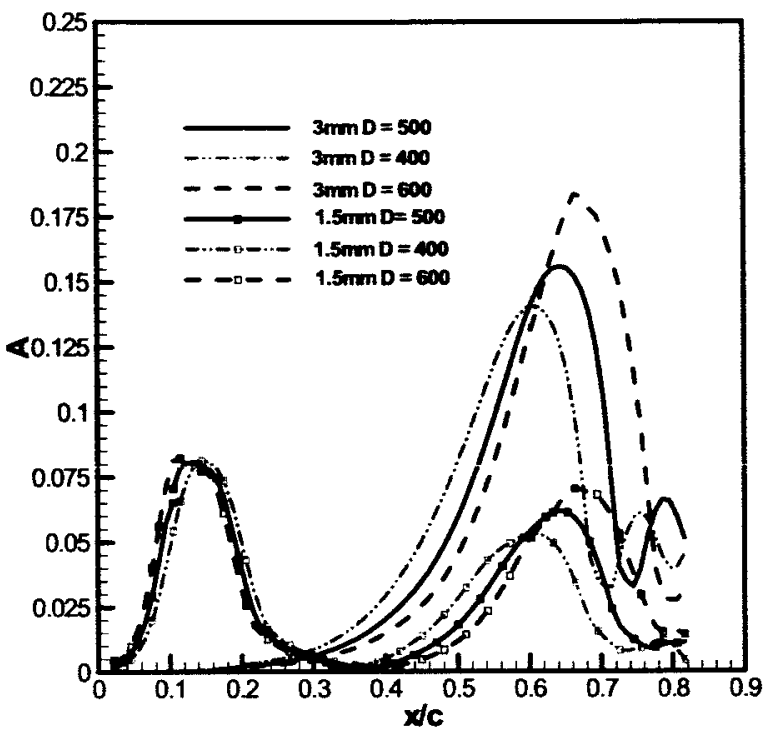

Figure 17: Effects of roughness dot size on the controlled $1.5 \mathrm{~mm}$ mode and the most unstable $3 \mathrm{~mm}$ mode. Initial amplitudes are computed by the adjoint PSE receptivity approach. 